\title{
Design and Demonstration of Intelligent Home Speech Recognition Control and Security System
}

\author{
Yaning Yan, Jing Li \\ School of Intelligence Science and Information Engineering \\ Xi'an PeiHua University, Xi'an, China \\ 394811297@qq.com, 285149439@qq.com
}

Keywords: MCU control; Speech recognition; Intelligence

\begin{abstract}
Computer automatic speech recognition and remote control technology is a major breakthrough in the realization of remote control intelligentization. This system is a 16 bit structure based on the Internet of things, which combines linear logic analysis technology, program-controlled switching, signal recognition, and micro controller design system based on user's instructions for simple word recovery. The user first sends out the voice information, the system transforms the voice information into the electrical signal, and then processes and analyses it, so as to achieve the purpose of controlling the home.
\end{abstract}

\section{Introduction}

The practical research of speech recognition system is a main direction of speech recognition research. Communicating with machine and letting machine understand what you say is a long dreamed thing for people. With the rapid development of microelectronic technology, computer technology and sensor technology, nowadays voice control technology has been applied to every corner of the society, providing people with various conveniences[1].

(1) Voice home: further improve the system's noise and recognition accuracy, and use the existing software to develop a unique voice chip, and integrate it on the remote control to make voice control home.

(2) Can identify the owner of the watchdog: on the basis of this system to expand the speaker recognition function, and the software hardware, integrated on the chip. The chip is arranged in the anti-theft door, which can complete the master function namely the open door.

(3) Sound control system on real car: in the process of driving, the other operations that are inconvenient to be done by hand can be achieved by the sound control system[2]. This is very similar to our system. However, there may be a lot of noise in the actual car, so the filter noise becomes the most critical technology.

Sound control home technology is to control the home through related voice. The use of the "before, after, left, right, stop" five words as home instruction. In this paper, an intelligent remote control home system with voice recognition function is designed by using the Lingyang microcontroller. The main functions of this system include:

(1) The switch function of home is realized by simple I/O operation.

(2) In accordance with the voice features of SPCE061A, the function of voice control is realized by using the system's voice playing and speech recognition resources.

(3) In the course of walking, sound control changes the state of home movement.

\section{Demonstration of Voice Home Scheme}

\subsection{Speech Control Scheme}

The process of machine speech recognition and processing is basically consistent with the process of speech recognition and understanding[3]. The mainstream speech recognition technology is based 
on the basic theory of statistical pattern recognition. A complete speech recognition system can be roughly divided into 3 parts: speech feature extraction, acoustic model and pattern matching (recognition algorithm) and semantic understanding. The basic principles are shown in Figure 1.

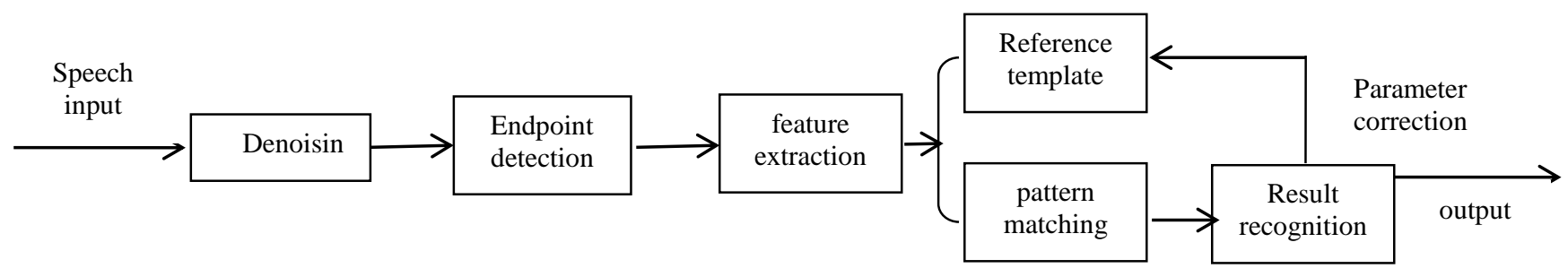

Figure 1. Principle block diagram of speech recognition system

We can see from the figure of speech recognition is generally divided into 2 steps: the first step is the system of "learning" or "training" stage, this stage of the task is the basic unit of the identification of the acoustic model and language model grammar analysis, namely the construction of library reference model; the second step is to "identify" or "the test stage, according to the type of recognition system can meet the requirements of the selection of recognition method, using speech analysis method to analyze the recognition method of speech feature parameters required by the comparison, according to certain criteria and measure the reference mode and model in the library, through the decision results.

The basic structure of speech recognition system includes preprocessing, A /D conversion, start and stop point recognition, feature extraction, recognition and judgement. The structure is shown in Figure 2 below.

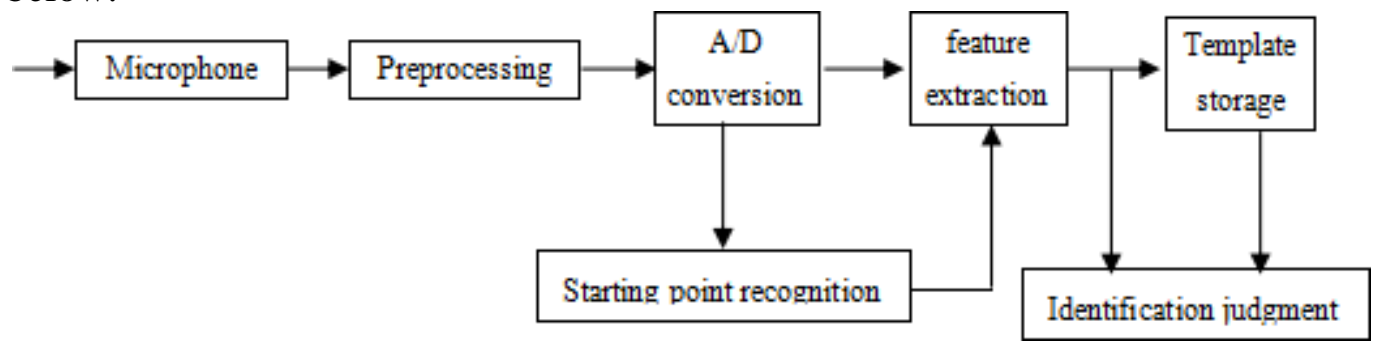

Figure 2. The basic structure of the speech recognition system

\subsection{Scheme Demonstration}

Speech recognition can be divided into isolated word recognition and continuous speech recognition, specific person recognition and non specific human recognition, according to the actual needs and the different applications. The main indicators of speech recognition are high recognition rate, real-time and large vocabulary. For a speech recognition system, we should also consider the characteristics of simple hardware and software design, low price, flexible peripheral control and convenient man-machine interaction. Now the chips used for speech recognition are mainly DSP (digital signal processor) chips, such as the TMS320 series of the TI company. However, it is obvious that DSP chips are used in small voice recognition systems. First, because of the pin, the price is expensive, cumbersome to use, the control function is weak, often need to be combined with the MCU or FPGA, to achieve human-computer interaction; secondly, often need external A /D conversion chip; third pin, $3.3 \mathrm{~V}$, FPGA, Flash, connected with the chip memory, to consider the level matching. These will make the whole system structure huge and the design is tedious.

MCS-51 Series MCU is used to realize speech recognition and voice playback. Therefore, speech recognition module and voice playback module need to be extended, which will inevitably cause the resource shortage of ports, so interface expansion chip must also be added[4]. The implementation scheme is shown in Figure 3. 


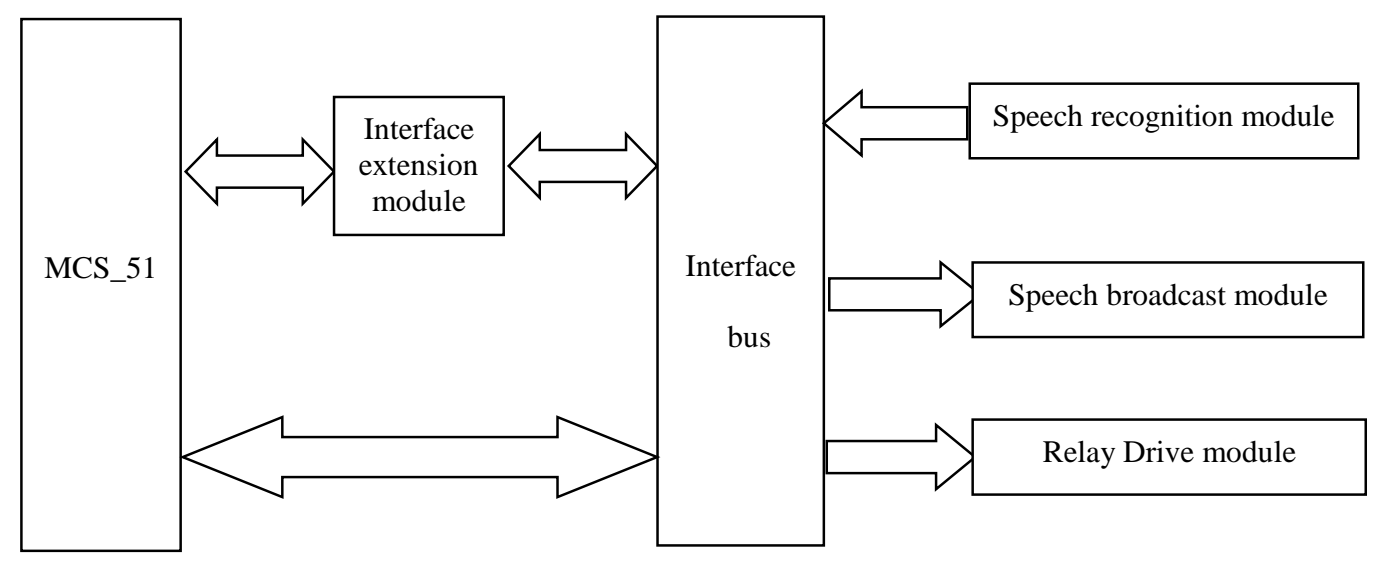

Figure 3. Using MCS-51 series singlechip to realize voice control home

SPCE061A is a micro controller of 16 bit structure launched by Lingyang technology. The CPU clock frequency is $0.32 \sim 49.152 \mathrm{MHz}$ and has high processing speed. It is a more economical choice in the digital speech recognition application field.3) Classroom: each classroom should have its own number and category (such as ordinary classrooms, multimedia classrooms, computer rooms, etc.), there is only one course held in one classroom during each period, and the type and capacity of the class must meet with the requirement the course at the same time.As shown in Figure 4

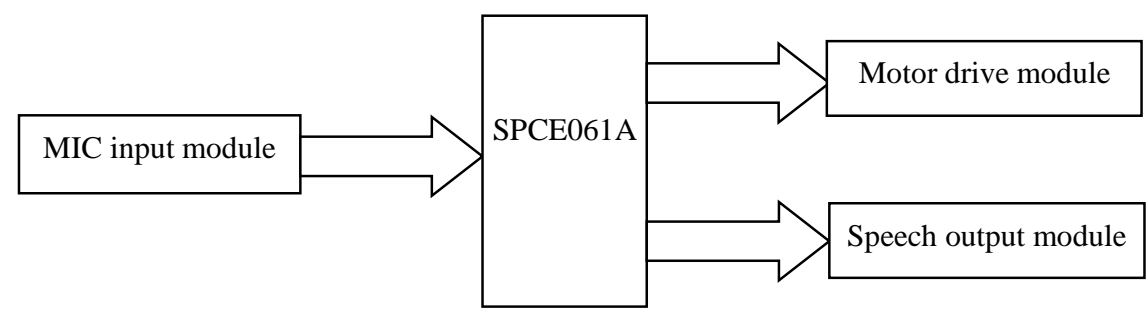

Figure 4. Home implementation of voice control based on SPCE061A

This design uses SPCE061A to realize the voice control home program. The system structure block diagram Figure 5 is as follows:

The system consists of two parts: the SPCE061A simplification development board and the voice home control circuit board. The speech input part MIC_ IN, keypad input KEY, voice output part of the power amplification link has been streamlined the development board 61 board, which has provided great convenience for our use. In terms of electrical drive, the full bridge driving technology is adopted, and four I/O ports are used to divide into two groups, which realize two electrical appliances' normal, reverse and stop three state operation.

\subsection{System Control Scheme}

Home control is controlled by voice control and interrupt timing control. After speech triggering household electrical appliances action and household electrical appliances action, the movement state of household can be changed at any time through voice commands[5]. When each action is triggered, the timer is started. If the home cannot receive voice instructions for some reasons, the interrupt service routine will issue instructions to stop the home as long as the time is up. 


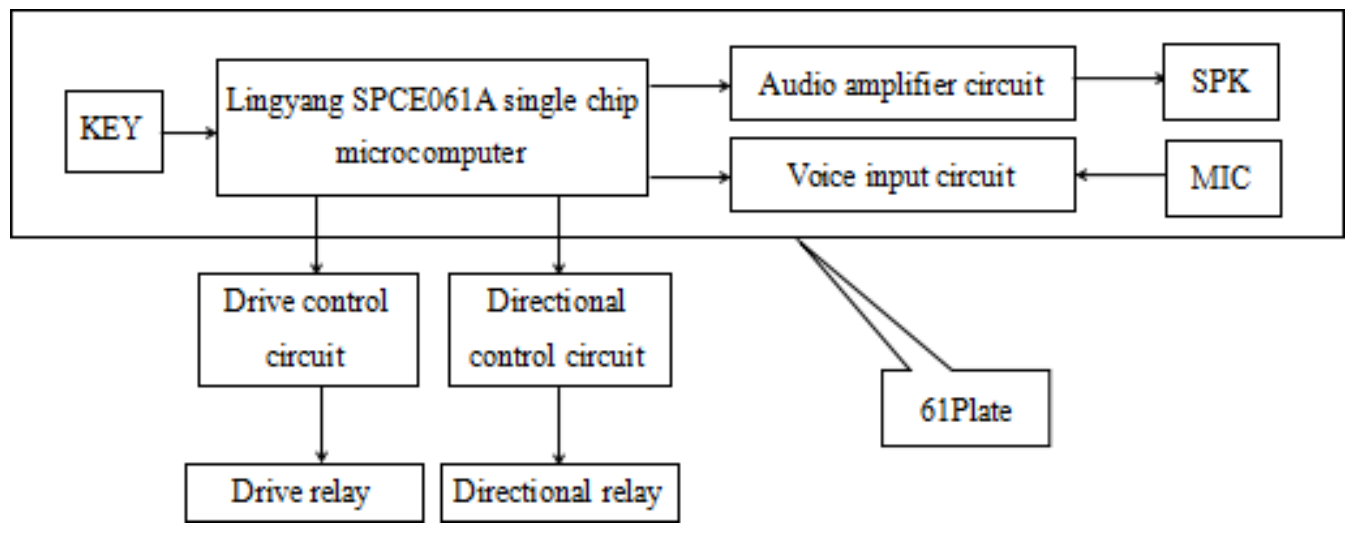

Figure 5.Structure system diagram

\section{System Design}

\subsection{Hardware Design}

The hardware part of the system is realized on the 61 board because most of its functions are completed. Only the electrical control part is designed on another independent circuit board. We call it the control panel. The overall design block diagram of the system is Figure 6 as follows.

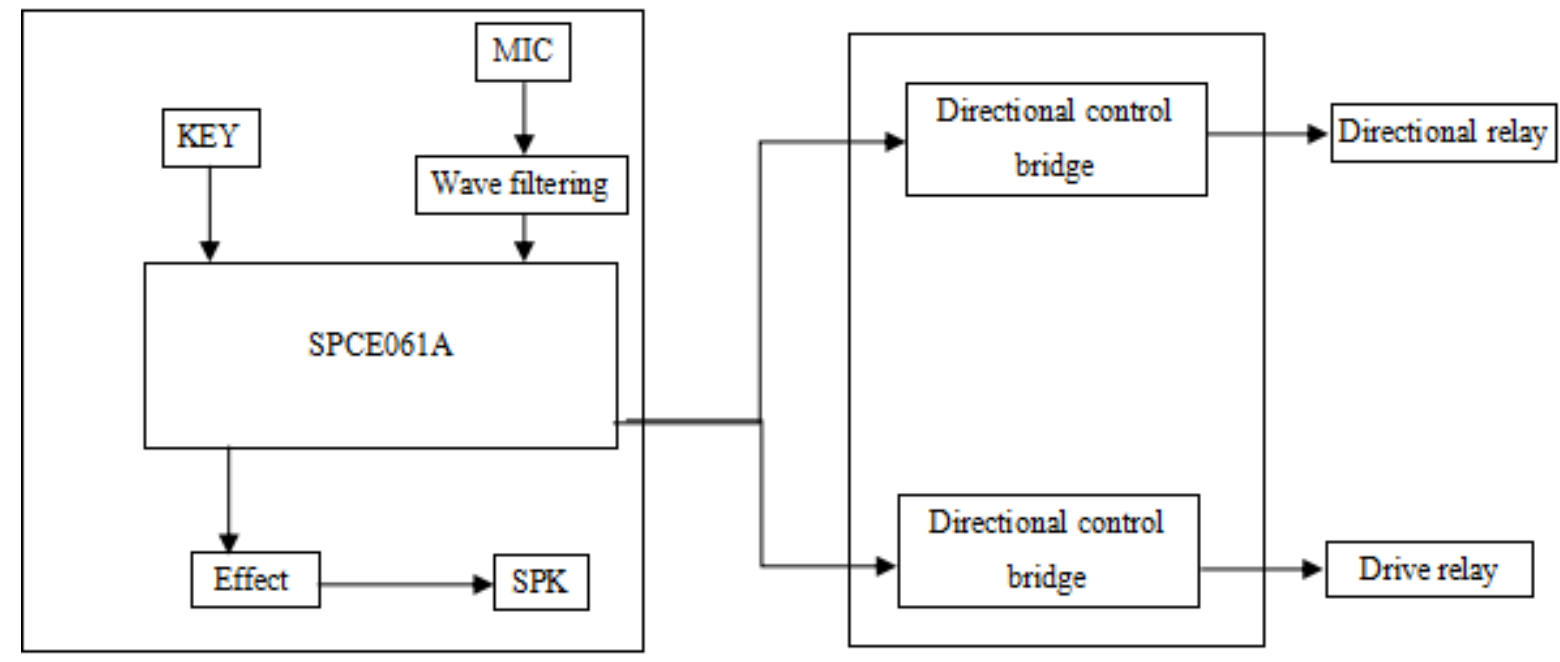

Figure 6. Block diagram of hardware overall design

\subsection{Software Design}

The main program flow of speech recognition home is divided into four parts, as shown in Figure 7: initialization, training, recognition, and training[6].

Initialization part: the initialization operation sets the IOB8 IOB11 to the output end to control the electrical appliance. If necessary, there should be a corresponding input and PWM port settings.

Training part: the work done in the training part is to build a voice model. The program begins to judge whether Home Furnishing trained, if no training is required to train the model, and will store the training to FLASH in training success, do not need re training in the future use; if you have trained the storage model in FLASH out loaded to the identifier.

Recognition part: in the identification link, if the identification result is a name, stop the current action and enter the standby state, and wait for the action command. If the identification result is 
action, the instruction home will inform and execute the speech by voice. During the movement, the home can be stopped by calling the name of the home.

Retraining operation: taking into account the re training needs, set up a re training button (61 in KEY3), the scan button, once detected button will erase training flag (0xe000 unit), and wait for the reset; reset program re execution, when the detected training flag 0xFFFF will be required for the re training.

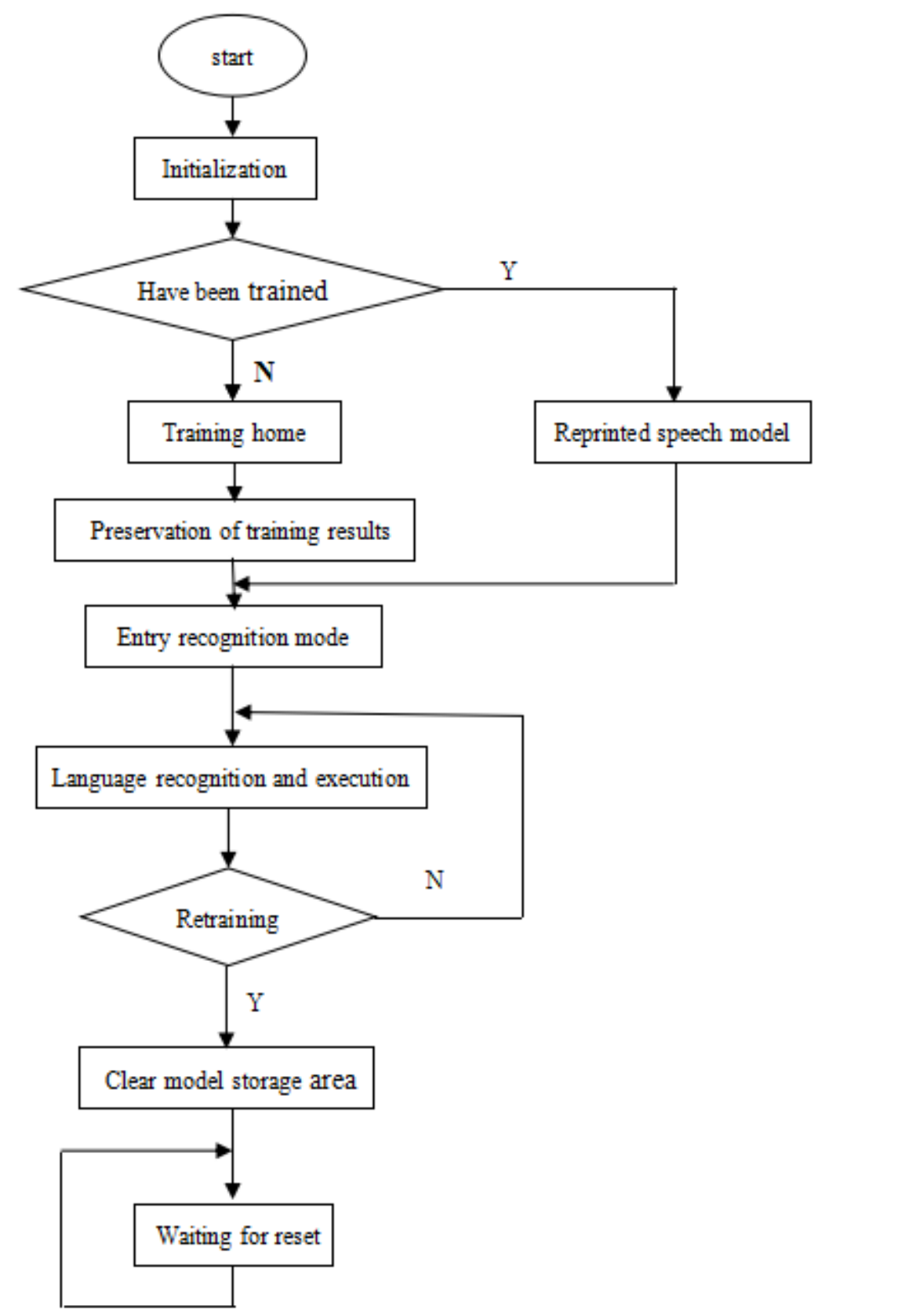

Figure 7. System general program flow chart

\subsection{Hardware Connection Debugging}

The voice control home circuit consists of two parts: the 61 board part and the control board part. The hardware part is connected mainly to the IOB8 IOB11 of SPCE061A to control the state of the home. The power supply is connected, the battery power is directly connected with the control board 
power interface $\mathrm{J} 1$, and the $\mathrm{J} 2$ is connected to the power supply of the 61 board, and the power polarity is paid attention to when the connection is connected.

There are two cases of I/O connection between the 61 board and the control board:

The I/O pin down 61 plates, 61 directly to the board and control board can be buckled together, 61 plates connected by J6 and IOBH control circuit;

Using I/O pin to the 61 board, the direct use of cable 61 board IOBH and J5 can be connected.

\section{Conclusion}

Intelligent Home Furnishing system networking can provide intelligent control scheme based on the people in the future will be able to experience and enjoy the beauty of nature in position, while increasing the intensity of dealing with emergencies, enhance the sense of security in no home, so that people can full physical and mental work, so as to improve the quality of life, and promote the residential district intelligent construction process.

\section{Acknowledgment}

This work is supported by the university level research fund of Xi'an Peihua University (Grant No.PHKT17080).

\section{References}

[1] Zhou Liuping. 2016. Design manual for industrial and civil power supply and distribution[D]. Beijing, China Power Publishing House.

[2] Gutierrez et al, Wang Quan et al. 2015. LR-WPAN: implementation of wireless sensor network based on 802.15.4 IEEE. Beijing: Mechanical Industry Press.

[3] Wang Center, Wang Dongfang, Wang Yawei, et al. 2016. Based on LD3320 speech recognition module, the intelligent home acoustic control system [J]. Internet of things technology, 6 (11): 19-21.

[4] He Kan, Tian Yaqing, Li Qiang, et al. 2015. Based on LD3320, speech recognition intelligent trash can design $[\mathrm{J}]$. foreign electronic measurement technology, (6): 85-88.

[5] Ke Guoqin. 2011. Design of intelligent home system based on single chip microcomputer [J]. Journal of Chifeng University (NATURAL SCIENCE EDITION).

[6]Yang Xi, Zhang Wenzhao, Liang Xiaolin. 2016. Application of speech recognition in smart home control system [J]. Journal of Hunan University of Science and Engineering, 37 (10): 34-35. 